\title{
Learning data structure from classes: A case study applied to population genetics
}

\author{
J.J. del Coz ${ }^{\mathrm{a}, *}$, J. Díez ${ }^{\mathrm{a}}$, A. Bahamonde ${ }^{\mathrm{a}}$, F. Goyache ${ }^{\mathrm{b}}$ \\ a Artificial Intelligence Center, University of Oviedo at Gijón, 33271 Asturias, Spain \\ b SERIDA-Deva, Camino de Rioseco, Gijón, Asturias, Spain
}

\section{A R T I C L E I N F O}

\section{Article history:}

Received 4 November 2010

Received in revised form 24 November 2011

Accepted 28 December 2011

Available online 3 January 2012

\section{Keywords:}

Clustering

Hierarchical classification

Pairwise classification

\begin{abstract}
A B S T R A C T
In most cases, the main goal of machine learning and data mining applications is to obtain good classifiers. However, final users, for instance researchers in other fields, sometimes prefer to infer new knowledge about their domain that may be useful to confirm or reject their hypotheses. This paper presents a learning method that works along these lines, in addition to reporting three interesting applications in the field of population genetics in which the aim is to discover relationships between species or breeds according to their genotypes. The proposed method has two steps: first it builds a hierarchical clustering of the set of classes and then a hierarchical classifier is learned. Both models can be analyzed by experts to extract useful information about their domain. In addition, we propose a new method for learning the hierarchical classifier. By means of a voting scheme employing pairwise binary models constrained by the hierarchical structure, the proposed classifier is computationally more efficient than previous approaches while improving on their performance.
\end{abstract}

(C) 2012 Elsevier Inc. All rights reserved.

\section{Introduction}

In many machine learning and data mining applications, users are not only interested in learning accurate classifiers or predictors, but also in gaining some insight into the application domain. This is the reason why more easily interpretable models are sometimes preferred, like those represented by decision trees or rules, over others with a better predictive accuracy, but which are less explanatory.

In this respect, Husymans et al. pointed out [23] that little research has been done to increase model interpretability for end-users, mainly due to the subjective nature of said interpretability. Comprehensibility usually depends on some factors outside the model, such as the user's prior knowledge and experience. However, some representations are generally considered to be more easily interpretable than others. In [18], the authors present a critical review of different knowledge representations particularly suitable for discovering comprehensible knowledge in the context of protein function prediction. Barros et al. [3] introduce a learning method aimed at providing a trade-off between predictive performance and comprehensibility, helping end-users to acquire new insights, confirming or rejecting their previously formed hypotheses. All these recent papers show that acquiring comprehensible knowledge is, and will be, a key issue and a challenge in learning from data.

\footnotetext{
* Corresponding author.

E-mail addresses: juanjo@aic.uniovi.es (J.J. del Coz), jdiez@aic.uniovi.es (J. Díez), antonio@aic.uniovi.es (A. Bahamonde), fgoyache@serida.org (F. Goyache)

URL: http://www.aic.uniovi.es (J.J. del Coz).
} 
In many applications the unique goal is actually to extract new knowledge; this is even more usual when some unsupervised learning techniques are applied. Among methods of this kind, clustering [21] stands out as one of the most prominent, allowing groups of homogeneous objects to be automatically obtained according to a target similarity measure. Practitioners can then analyze the properties that make each group different from the rest, obtaining valuable information about the underlying structure of the data.

There is a wide range of clustering algorithms, but some of them are more informative than others, such as those that not only output the set of groups, but also express their relationships, for instance, through a graph. This is the case of agglomerative hierarchical clustering methods [22,25,34]. These algorithms work in a bottom-up fashion, starting out by considering each instance as a cluster, and iteratively combining the two most similar clusters in terms of a similarity function. The merging process is repeated until all individuals form a single cluster. The hierarchical clustering output is usually described by a dendrogram, the main advantage of which is that it represents a nested tree of partitions and is more informative than non-hierarchical clusterings. This paper presents a new agglomerative hierarchical clustering algorithm. The novelty of our method is that instead of grouping individual objects, it is able to cluster sets of individuals. The key element is a metric that allow us to measure how similar two sets of objects are.

From a multi-class learning task, in addition to a classifier, it is also possible to infer some useful knowledge about the relationship between the classes involved. This is the case of applications in the field of population genetics, which constitute the underlying motivation for this research study. Such applications usually deal with some kind of genetic information on several individuals that belong to a set of populations (e.g. different species or breeds). The main goal is to discover relationships between these populations according to the genetic descriptions of their individuals. For instance, in [29], nine Iberian roe deer (Capreolus capreolus) populations were analyzed in order to study their genetic variability and differentiation; in [13], 618 individuals from seventeen British populations of the Eurasian otter (Lutra lutra) were used to infer patterns of gene flow in Scottish otters and assess the influence of fragmentation on the genetic structure of otters in Wales and South West England; while [20] studied fifty indigenous cattle breeds from Africa together with five European (Bos taurus) and three Asian (Bos indicus) breeds to identify genetic signatures of their origins, spread and differentiation. In this paper, we address these three interesting applications to show that the proposed method can prove very useful in this context.

The main tool used by practitioners to solve these problems is a clustering algorithm, called Structure [27], that uses multi-locus genotype data to investigate population structure. It is usually employed to infer the presence of distinct populations, assigning individuals to populations and estimating population allele frequencies. However, its main drawback for the aforementioned applications is that it clusters individuals instead of classes. Users thus analyze the groups so obtained in order to infer the relationship between these populations. Using this kind of clustering method, or other algorithms for clustering examples, knowledge discovery depends heavily on the capacity of users to interpret the clusters of individual examples. Here we propose a different approach, namely that of directly clustering the set of classes: in applications of this kind, the set of populations.

In recent years, some authors [5,26,30,32] have proposed decomposition algorithms to solve multi-class tasks. The underlying idea in all these approaches is the same: to learn a binary decision tree classifier based on a hierarchy of classes. These methods first build a dendrogram of classes and then a binary Support Vector Machine (SVM) classifier [31] is learned for each internal node of that hierarchy in order to separate the examples of each subset of classes. The classification procedure proceeds from the root to the leaves guided by the predictions of SVM classifiers at internal nodes. All these methods differ basically in the way they build the dendrogram of classes. For instance, Dendrogram-based Support Vector Machines (DSVM) [5] measure the similarity of each pair of classes using the Euclidean distance between their centroids. A top-down recursive method, called Divide-by-2 (DB2), is presented in [32] that employs a $k$-means clustering of the centroids of classes to iteratively divide the set of classes into two groups.

However, the most relevant work is probably that of [30], which presents a multi-class classifier for high-dimensional input spaces, called the Margin Trees (MT) classifier. In this case, in order to build the dendrogram of classes, Tibshirani and Hastie propose using the pairwise margins to compute the distance between each pair of classes so as to then apply an agglomerative hierarchical clustering using a complete linkage procedure. They found that the MT classifier had an accuracy that could compete with a one-vs-one multi-class SVM [11,24,31] and nearest centroids on seven cancer microarray data sets. Despite the fact that these authors are more interested in the method as a classifier, they remark on the additional interpretability of the model obtained in biological tasks; i.e., they point out the utility of the cluster of classes in real applications.

Since their method is restricted to those multi-class problems in which every pair of classes are linearly separable, they apply a hard margin approach. The so-called Soft Margin Trees (SMT) classifier is presented in [16]. This is a generalization of the MT method to the non-separable case, applying the basic principles of margin maximization. Said paper also reports an extensive experimental study that shows that the SMT classifier has significantly worse accuracy than multi-class SVM, but is still very useful to obtain a meaningful organization of the set of classes that will be easily interpreted by an expert in the domain.

In this paper we present a new method, Pairwise Soft Margin Trees, (PSMT) to improve both the computational efficiency and classification performance of the SMT algorithm: it achieves similar accuracy results to those obtained by the one-vs-one multi-class SVM and is faster than SMT. We also propose the use of PSMT in population genetics applications to obtain: (1) a hierarchical clustering of the set of populations, i.e., a binary tree or dendrogram with classes placed at leaves and (2) a hierarchical classifier $[7,28,17]$ that can discriminate samples from said populations. Both the dendrogram and the hierarchical 
classifier can be broken up at different levels to yield different clusterings of the set of classes and different classifiers. As we shall describe in Section 3, these classifiers can be viewed as non-deterministic [10] or set-valued classifiers [9] that assign a group of classes instead of a concrete class. This is also related to classifiers with a reject option [4,8], which have been used in some applications [1].

The paper is organized as follows. In Section 2, we describe the SMT and PSMT algorithms and highlight the differences between both approaches. Section 3 is devoted to explaining how practitioners can interpret the different outputs of PSMT to extract valuable information about their domain. Section 4.1 then provides a comparison between the method proposed in this paper and the original SMT. In Section 4.2, we describe the general setting used to solve population genetics tasks and the experimental results obtained in the three applications described previously. We shall show that our approach can be very useful in this context. We close the paper with a number of conclusions.

\section{Soft Margin Trees}

Here we briefly describe the SMT classifier as the method used to address population genetics applications. A more indepth discussion of SMT and an experimental study can be found in [16].

Let $\mathcal{X}$ be an input space and $\mathcal{Y}=\left\{C_{1}, \ldots, C_{N}\right\}$, a finite set of classes. We consider a multi-class classification task given by a training set $S=\left\{\left(\vec{x}_{1}, y_{1}\right), \ldots,\left(\vec{x}_{n}, y_{n}\right)\right\}$ drawn from an unknown distribution $\operatorname{Pr}(X, Y)$ from the product $\mathcal{X} \times \mathcal{Y}$. Within this context, our learning task is to build a dendrogram (or hierarchical clustering), $T$, in which each class labels exactly one leaf; $T$ has $N$ leaves and $N-1$ internal nodes. Figs. 2, 5 and 7 show the dendrograms obtained by applying the method discussed here to the data sets described in Section 4.2.

In order to build these dendrograms, our agglomerative hierarchical clustering method begins with each class as a separate cluster and merges them into successively larger clusters. At each step, the two most similar clusters are merged; thus similarity or distance functions play a major role in this method. SMT calculates a symmetric dissimilarity matrix, $D$, based on pairwise dissimilarities or distances and then applies a complete linkage procedure. The value in the $l$ th row, $m$ th column is the distance between classes $C_{l}$ and $C_{m}$. The idea underlying SMT is that the distance between two classes depends on a binary SVM classifier trained with the examples of both classes. Notice that it will be necessary to learn $\left(\begin{array}{c}N \\ 2\end{array}\right)$ binary SVMs to calculate $D$. Once the hierarchical clustering of classes is obtained, a hierarchical classifier is learned using a set of binary classifiers, one for each internal node.

In the MT algorithm [30], the distance between two separable classes is defined as the margin between them. To compute the distance between classes $C_{l}$ and $C_{m}$, the class labels $\left(y_{i}\right)$ of the examples of these classes are relabeled $\left(y_{i}^{\prime}\right)$ as +1 and -1 , respectively. The hyperplane of maximum margin that separates the nearest examples of these classes is defined by a weight vector, $\vec{w}$, and a bias, $b$, that can be obtained by solving the following optimization problem:

$$
\begin{aligned}
& (\vec{w}, b)=\arg \max _{\|\vec{w}\|=1}(M), \\
& \text { s.t. } \quad y_{i}^{\prime}\left(\left\langle\vec{w}, \vec{x}_{i}\right\rangle+b\right) \geqslant M, \quad \forall y_{i} \in C_{l} \cup C_{m} .
\end{aligned}
$$

Finally, in MT the distance between classes $C_{l}$ and $C_{m}$ is the margin:

$$
D(l, m)=2 \cdot M \text {. }
$$

Actually, the optimization problem in Eq. (1) is equivalent (see for instance [6,12]) to a typical hard-margin SVM formulation:

$$
\begin{array}{ll}
\min & \frac{1}{2}\|\vec{w}\|^{2}, \\
\text { s.t. } & y_{i}^{\prime}\left(\left\langle\vec{w}, \vec{x}_{i}\right\rangle+b\right) \geqslant 1, \quad \forall y_{i} \in C_{l} \cup C_{m} .
\end{array}
$$

In this case, the margin between both classes is equal to:

$$
D(l, m)=\frac{2}{\|\vec{w}\|} .
$$

The approach followed by SMT is to use a soft-margin SVM formulation instead of a hard-margin formulation:

$$
\begin{aligned}
& \min \frac{1}{2}\|\vec{w}\|^{2}+C \sum_{\forall y_{i} \in C_{l} \cup C_{m}} \xi_{i}, \\
& \text { s.t. } \quad y_{i}^{\prime}\left(\left\langle\vec{w}, \vec{x}_{i}\right\rangle+b\right) \geqslant 1-\xi_{i}, \\
& \xi_{i} \geqslant 0, \quad \forall y_{i} \in C_{l} \cup C_{m},
\end{aligned}
$$

where $C$ is a regularization parameter. The distance between classes $C_{l}$ and $C_{m}$ is defined by the following expression:

$$
D(l, m)=\frac{1}{\frac{1}{2}\|\vec{w}\|^{2}+C \sum_{\forall y_{i} \in C_{l} \cup C_{m}} \xi_{i}} .
$$




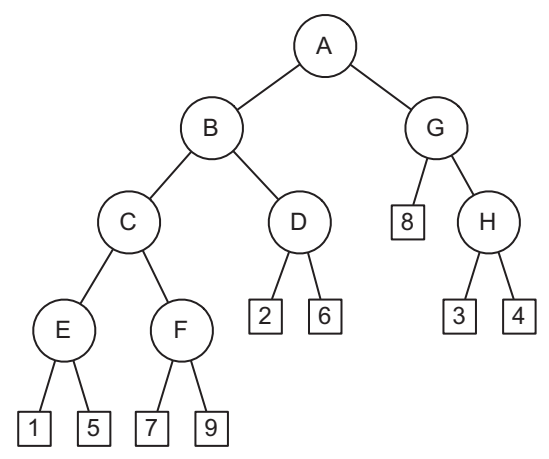

Fig. 1. Example of a hierarchy. This is the tree representation of the Roe Deer dendrogram depicted in Fig. 2 . There are nine classes and eight internal nodes. If we want to decide whether an instance goes down from B to the left or to the right, PSMT evaluates that instance with the pairwise models that discriminate a class belonging to the left branch from a class belonging to the right branch.

The idea is that when classes are very different, the classifier will be given by a simple model (i.e., $\|\vec{w}\|$ will be low) and/or the number of misclassified examples or points inside the margin will be small $\left(\sum \xi_{i} \rightarrow 0\right)$, and hence the distance (Eq. (6)) will be high. When classes are similar, the model will be complex (the norm of the weight vector will be high) and/or there will be a lot of misclassified examples or points inside the margin $\left(\sum \xi_{i} \gg 0\right)$, in which case, the distance will be small. Thus, the optimization problem in Eq. (5) minimizes an expression that faithfully captures the differences between two classes.

It should be noted that, in order to ensure that all the distances in matrix $D$ share an identical scale, the regularization parameter $C$ must be the same in the $\left(\begin{array}{l}N \\ 2\end{array}\right)$ SVM binary classifiers needed to calculate all the pairwise distances.

\subsection{Improving the classification stage}

A straightforward approach to constructing a hierarchical classifier may consist in learning a family of models $\left\{\vec{w}_{1}, \ldots, \vec{w}_{(N-1)+N}\right\}$, one for each node of the tree. The task of these models is to decide whether an instance belongs to the class attached to the node. Then, an entry $\vec{x}$ will be assigned to all classes $j$ such that $\left(+1=\operatorname{sign}\left(\left\langle\vec{w}_{j}, \vec{x}\right\rangle\right)\right)$. ${ }^{1}$ However, this procedure may lead to inconsistent predictions with respect to the tree. To avoid such inconsistencies, a top-down prediction procedure can be used $[7,17,28]$.

The SMT algorithm learns a binary model for each internal node in the hierarchy $\left(\left\{\vec{w}_{1}, \ldots, \vec{w}_{N-1}\right\}\right)$. The task of these models is to separate classes belonging to the right branch from classes belonging to the left branch. So, for instance (see Fig. 1), for internal node B a binary model is learnt in which examples from classes 1, 5, 7 and 9 belong to the positive class and examples from classes 2 and 6 belong to the negative class (examples from classes 3, 4 and 8 are not used to obtain this model). Then, to classify a new instance we only have to descend in the hierarchy in the direction indicated by the model attached to each internal node until reaching a leaf. This method is faster than the previous one because it needs a lower number of models and not all the examples take part in the learning process of these models [16].

It is, however, possible to construct a hierarchical classifier without learning any new model for the internal nodes. We propose to follow an ensemble approach, reusing all the pairwise classifiers (Eq. (5)) learned to compute the distance between each pair of classes. Applying these pairwise models, we can classify new instances following a voting scheme in which only the models that involve a pair of classes that are not placed in the same branch of the subtree take part. For instance, to decide whether an example goes down through the left or the right branch from internal node B in Fig. 1, we take into account all the pairwise classifiers between a class of the left branch vs a class of the right branch; i.e., 1 vs 2,1 vs 6,5 vs 2,5 vs 6,7 vs 2,7 vs 6,9 vs 2 , and 9 vs 6 . The question now is how to combine the predictions of all these pairwise models.

\subsection{Decision rule}

We considered three different decision rules in order to select the winner branch, using the predictions of the pairwise classifiers yielded by the one-vs-one approach in all cases:

- Rule 1: The branch with the most wins.

- Rule 2: The branch of the class with the most wins.

- Rule 3: The branch of the class with the best percentage of wins.

\footnotetext{
${ }^{1}$ For ease of reading, we omit the threshold terms $b_{j}$; they can, however, be easily included by adding an additional feature of constant value to each $\vec{x}_{i}$.
} 
We also applied these rules with different tiebreak rules. The results of most of the combinations for benchmark datasets (see Section 4) were quite similar. The reason is that, ultimately, all decision rules aggregate the same models (the classifiers that also uses one-vs-one SVM). It is difficult for a particular decision rule to always be able to outperform the others. In most cases the differences were very small. The only exception was the second rule, which was sometimes significantly worse than the other two rules when the obtained dendrograms were very unbalanced, because it always benefits the branch with fewer classes. We then tested rules 1 and 3 with population genetics datasets (see Section 4.2 ) and found that the results were quite similar, although the third rule obtained slightly better scores when the number of clusters was higher.

Thus, the decision rule of PSMT works as follows. The winner is the branch of the class with the best percentage of wins. In the case of a tie between two classes, the winner is the branch of the class that appears in more pairwise classifiers. For instance, if we have a tie between two classes: class L (with 2 wins out of 3,66\%) and class M (with 4 wins out of 6 , $66 \%)$, the winner is the branch of class M. In other words, the branch with fewer classes. Finally, if the tie persists, the winner branch is selected at random.

The main idea of PSMT is to combine pairwise classifiers, eliminating the need to learn additional node classifiers. The resulting method is computationally cheaper, because the pairwise models must be computed before obtaining the hierarchical clustering of classes. However, the short study discussed above shows that several decision rules can be applied. In fact, there are other interesting alternatives, for instance using the cumulative margins $[7,28,14]$ instead of the predictions of pairwise classifiers.

\section{Interpreting the outputs of PSMT}

The aim of this section is to explain how practitioners can interpret the different outputs of Pairwise Soft Margin Trees. The most important result generated by PSMT is the hierarchical clustering of classes. End-users are usually familiar with this very common representation for clustering. For instance, in Fig. 2 we can see the clustering obtained with the Roe Deer data set (see Section 4.2.1). Notice that the hierarchical clustering can be broken up at different levels, thus obtaining different partitions. Practitioners can analyze those groups to infer new knowledge or to confirm their hypotheses. An adaptation
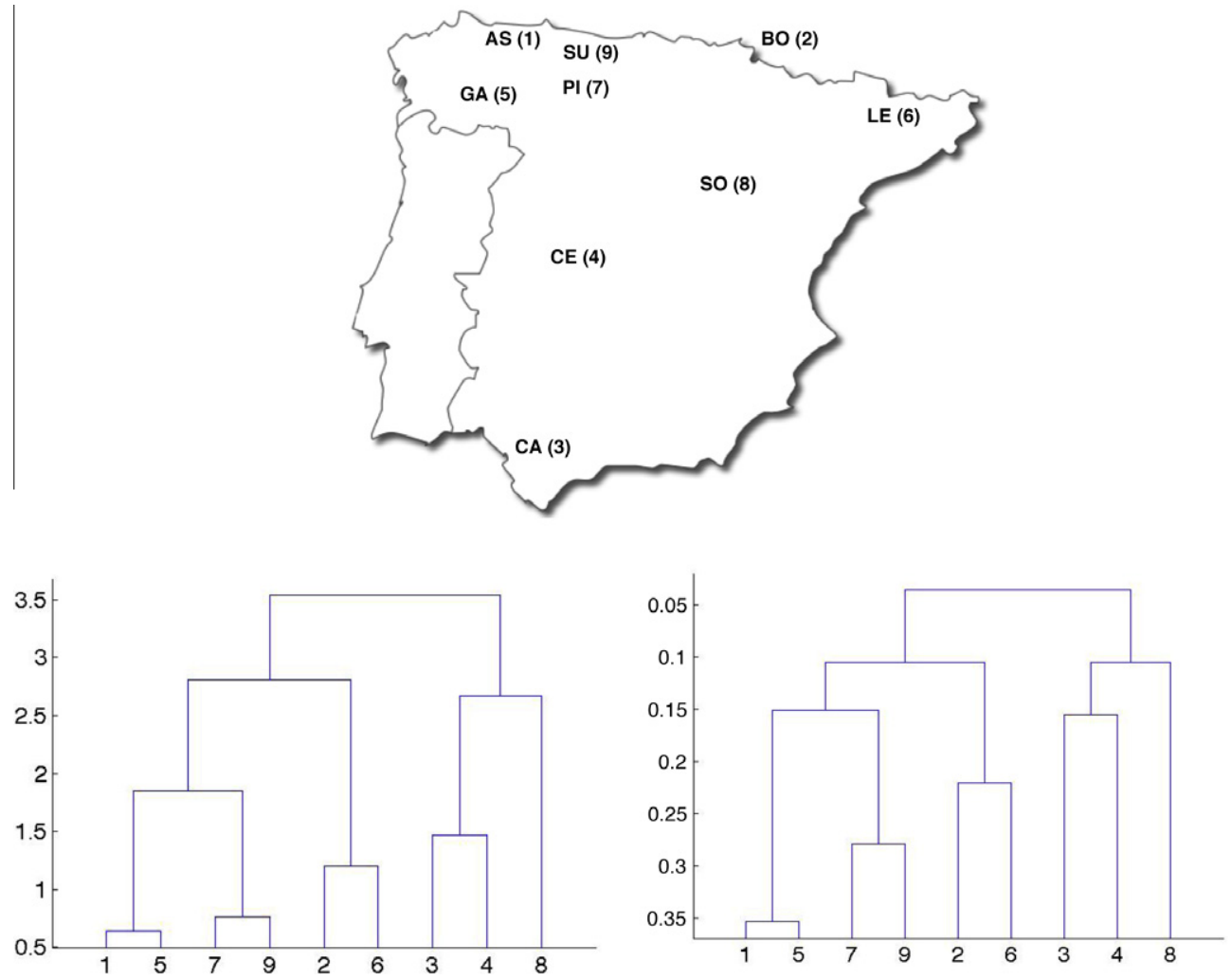

Fig. 2. Top. Geographical distribution of populations in the Roe Deer data set: (1) Western Asturias (AS), (2) Bordeaux (BO), (3) Cadiz (CA), (4) Central Spain (CE), (5) Galicia (GA), (6) Lleida (LE), (7) Picos de Europa (PI), (8) Soria (SO), and (9) Sueve (SU). Bottom. In the hierarchical clustering on the left, the $y$-axis represents the distances applying a complete linkage method. In the chart on the right, these distances have been replaced by the $0 / 1$ error of the different cluster partitions. 

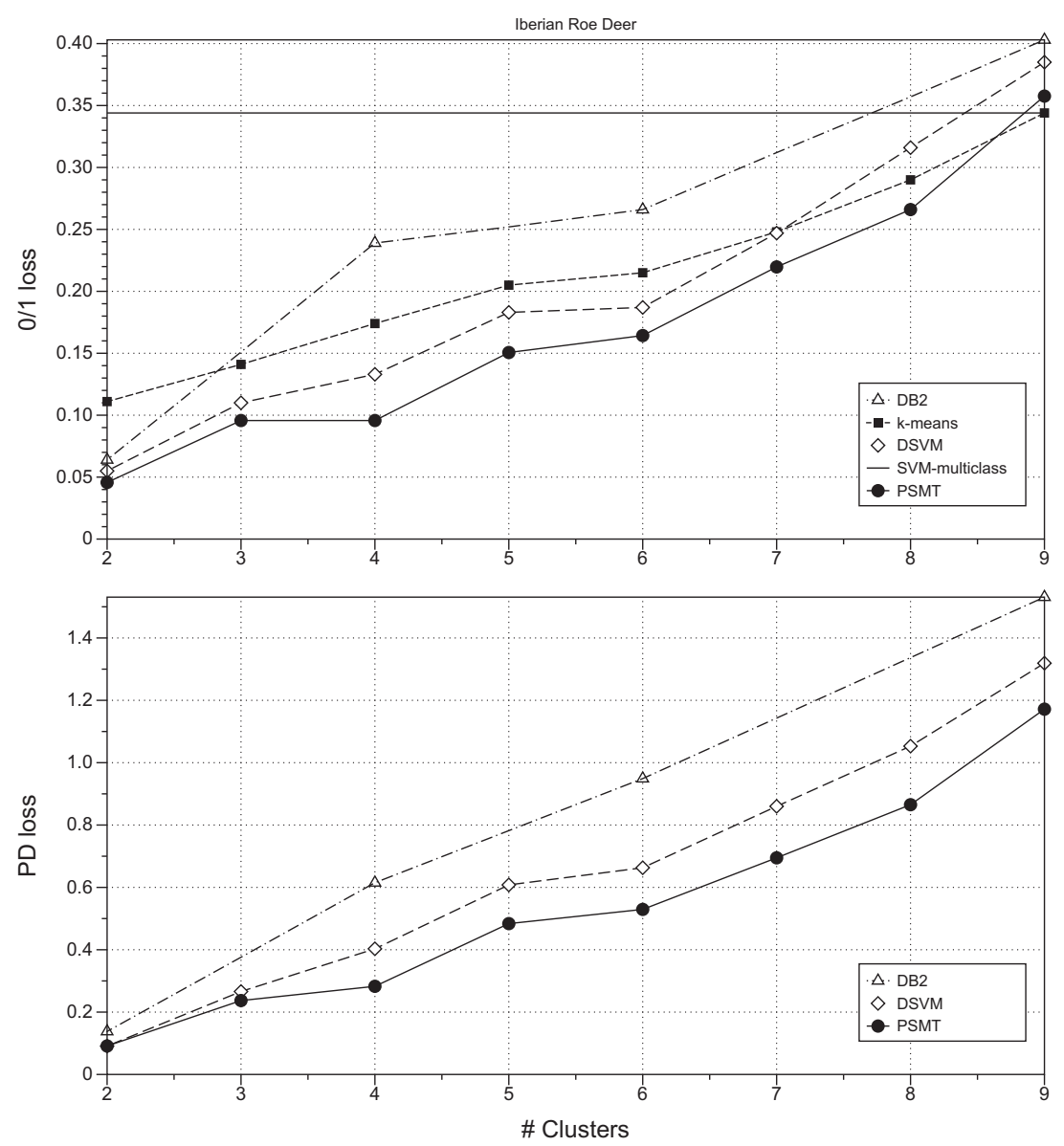

Fig. 3. Results in the Roe Deer data set. At the top, the graph of the $0 / 1$ loss for each number of clusters. At the bottom, the Prediction Distance loss only for the DSVM, PSMT and DB2 methods.

Table 1

Different clusters obtained in the Roe Deer data set. See Fig. 2.

\begin{tabular}{ll}
\hline Clusters $_{2}$ & $\{1,5,7,9,2,6\}$ and $\{3,4,8\}$ \\
Clusters $_{3}$ & $\{1,5,7,9\},\{2,6\}$ and $\{3,4,8\}$ \\
Clusters $_{4}$ & $\{1,5,7,9\},\{2,6\},\{3,4\}$ and $\{8\}$ \\
Clusters $_{5}$ & $\{1,5\},\{7,9\},\{2,6\},\{3,4\}$ and $\{8\}$ \\
Clusters $_{6}$ & $\{1,5\},\{7,9\},\{2,6\},\{3\},\{4\}$ and $\{8\}$ \\
Clusters $_{7}$ & $\{1,5\},\{7,9\},\{2\},\{6\},\{3\},\{4\}$ and $\{8\}$ \\
Clusters $_{8}$ & $\{1,5\},\{7\},\{9\},\{2\},\{6\},\{3\},\{4\}$ and $\{8\}$ \\
Clusters $_{9}$ & $\{1\},\{5\},\{7\},\{9\},\{2\},\{6\},\{3\},\{4\}$ and $\{8\}$ \\
\hline
\end{tabular}

of the F1 measure [10] could be used as a stop criterion in the splitting process aimed at selecting the best level at which to break up the hierarchical clustering.

However, not only the learned dendrogram can be broken up at different levels; the hierarchical classifier can also be broken up. For instance, in the Roe Deer data set, given the graph of the 0/1 loss (see Fig. 3), a practitioner can decide to break up the hierarchical clustering to obtain 4 different groups $\{1,5,7,9\},\{2,6\},\{3,4\}$ and $\{8\}$ (see Table 1 ). The $0 / 1$ loss is below $10 \%$. In order to obtain a classifier for this set of groups, we do not need the pairwise classifiers 1 vs 5,1 vs 7,1 vs 9,5 vs 7,5 vs 9 , 7 vs 9,2 vs 6 or 3 vs 4 . The final classifier rejects (does not use) these pairwise classifiers because they include pairs of classes that belong to the same cluster. Of course, the remaining pairwise classifiers are used to decide which of the four groups is predicted for a new instance.

The term reject is taken from classifiers with a reject option [4,8]. These classifiers are allowed to abstain from a prediction for a new query instance when they are not sufficiently sure. Here, we reject (do not use) all the predictions made by some pairwise classifiers. This is also related to set-valued [9] or non-deterministic classifiers [10], because after pruning or rejecting the pairwise models used to make a decision at an internal node of the dendrogram, the resulting hierarchical classifier can be seen as a classifier allowed to predict more than one class (a group of classes) for some entries in the input space. 
Applying this pruning process in the hierarchical order learned by the hierarchical clustering, we have a bunch of classifiers, $h_{k}: \mathcal{X} \rightarrow$ Clusters $_{k}$, one for each partition of $k$ clusters (see Table 1 ). In this case, we consider an example to be classified correctly if the cluster thus predicted contains its true class. We can accordingly obtain classification errors not only for the original set of $N$ classes, but also for $k=N-1, \ldots, 2$ number of clusters, without learning any new model. Fig. 3 shows the classification errors of the hierarchical classifier learned from the hierarchical clustering depicted in Fig. 2 for every possible number of clusters. Obviously, if we consider the 0/1 loss function, the classification error will decrease as we prune the original hierarchical classifier (as $k$ decreases).

This set of $0 / 1$ loss values obtained for each number of clusters can show very useful information and can be a good replacement for the $y$-axis distances of the hierarchical clustering, which are sometimes difficult to interpret. In a hierarchical clustering, the $y$-axis usually represents the distance between clusters (see Fig. 2, bottom-left). However, these distances are often not very informative, as they can depend on the linkage method employed. In PSMT, these distances can be replaced by the $0 / 1$ loss or the accuracy obtained by the set of $h_{k}$ classifiers (see Fig. 2, bottom-right). The hierarchical clustering chart thus shows the user not only all the partitions that can be made, but also how easy it is to discriminate between these groups.

Analyzing the results of PSMT over the Roe Deer data set, also depicted in Fig. 3 (top), the 0/1 loss for 4 clusters is quite small (less than 10\%) and even for 6 clusters, the error is acceptable (around 15\%). In fact, there is only a small increase in the $0 / 1$ error between 5 and 6 clusters. This means that classes 3 and 4 are easy to distinguish. For more than 6 clusters, the $0 / 1$ error increases almost linearly with the number of clusters. This means that classes 1 and 5, 7 and 9, and 2 and 6 are very difficult to distinguish. As they were sampled in close geographical or environmental areas, it is not surprising that our hierarchical clustering (see Fig. 2) identifies them as those populations with the highest genetic identity.

Another kind of information that can be useful to study is what happens when a hierarchical classifier fails. A hierarchical clustering of classes must somehow represent the similarity between these classes. When the hierarchical classifier with $k$ classes $\left(h_{k}\right)$ misclassifies an example $\vec{x}_{i}$, if the hierarchy is good, the predicted group of classes $h_{k}\left(\vec{x}_{i}\right)$ must be near (in the hierarchy) to the node containing the true class $y_{i}$. Therefore, we can compute the distance in the hierarchy between predictions and true classes; i.e., the number of arcs along the path between the nodes labeled by those classes in the dendrogram $T$. This measure was originally presented in the Hieron method of Dekel et al. [14] under the name of $\gamma$. In order to be more informative, we rename it as the Prediction Distance $(P D)$ :

$$
P D\left(h_{k}\left(\vec{x}_{i}\right), y_{i}, T\right)=\# \operatorname{arcs}\left(h_{k}\left(\vec{x}_{i}\right), y_{i}, T\right) .
$$

For instance, considering the $h_{8}$ classifier in Figs. 1 and 2, the $P D$ of an example from class 7 (see Fig. 2) classified as the group of classes $\{1,5\}$ is 3 , but if the prediction were class 2 , the $P D$ would increase to 5 . The furthest classes from class 7 are 3 and 4, both a distance of 7. Note that the maximum distance between two classes in a dendrogram of $N$ classes is $N$ and the minimum is 2 . Averaging this metric over the total number of test examples, we shall measure the goodness of a hierarchy $T$.

\section{Experimental results}

This section has two main objectives. The first (see Section 4.1) is to demonstrate that the PSMT algorithm is able to build meaningful hierarchical clusters of classes while improving the predictive power of SMT, thereby producing more accurate results comparable to those obtained with one-vs-one multi-class SVM. The second objective is to show how clustering-ofclasses algorithms can be used to deal with problems in which the main goal is to discover relationships between the classes involved (see Section 4.2).

In order to test our method, we employed six different learning algorithms in the experiments. Three of these represent classes by means of their centroids, i.e.:

$$
\vec{\mu}_{m}=\frac{1}{n_{m}} \sum_{\forall \vec{x}_{i} \in C_{m}} \vec{x}_{i}
$$

where $n_{m}$ is the number of data points in class $C_{m}$. We then tested different methods able to solve multi-class tasks with or without a hierarchy:

- SVM. A multi-class support vector machine based on one-vs-one models and the Max Wins [19] method to combine the output of binary models in the classification stage.

- DSVM [5]. Dendrogram-based Support Vector Machines build a dendrogram employing the distance between the different classes, which is computed by means of the Euclidean distance between the centroids of each pair of classes.

- $k$-Means. We run the k-means algorithm with $k=2, \ldots, N-1$, employing the centroids of the classes. A multi-class SVM classifier is then learned for each different group of clusters. We obtain classification results from 2 to $N-1$ clusters of populations.

- Divide-by-2 (DB2) [32]. This method recursively applies a k-means algorithm (with $k=2$ ) using the centroids of the classes to build a binary tree with the set of classes at its leaves. In this case, we cannot obtain $N-1$ different classifiers because this method does not produce a hierarchical clustering. The learned binary tree can only be broken up using the depth of its leaves. In line with the idea discussed in Section 3, we thus obtain a variable number of classifiers that depends on the topology of the tree. 
- Soft Margin Trees (SMT) [16]. The hierarchy of classes is constructed from the distance matrix computed by applying Eqs. (5) and (6). A binary model is then learnt at each internal node of the hierarchy to separate examples belonging to the two descending branches.

- Pairwise Soft Margin Trees (PSMT). The difference with respect to SMT is that it is not necessary to learn a new model at each internal node because the pairwise models learned to construct the hierarchy are also used to build the hierarchical classifier (see Section 2.1).

All the reported scores were estimated by means of a fivefold cross validation repeated twice. We did not use the ten-fold procedure, as in certain data sets there are too few examples in some classes. The SVM implementation used was libsvm [33], with the linear kernel for all methods. For each hierarchical classifier, DSVM, DB2 and SMT, we used the same regularization parameter $C$ in all models learned at each node in the tree. In the case of $\mathrm{k}$-means classifiers, we used a different value of $C$ for each of them. In all cases, we employed a twofold cross-validation (using only training data) repeated five times, searching within $C \in\left[10^{-2}, \ldots, 10^{2}\right]$ to select this parameter. The aim in this grid search was to optimize the $0 / 1$ loss of the original multi-class task. We report the error rate of these classifiers in addition to the Prediction Distance (PD) (Eq. (7)). Although the quality of the trees can be measured only by means of the Prediction Distance, we include the 0/1 los so as to compare their performance as classifiers with the multi-class SVM.

\subsection{Comparison with other algorithms}

We conducted a battery of experiments to analyze the improvement that the pairwise methodology (see Section 2.1) contributes to the idea presented in the SMT algorithm. The aim was to show that the PSMT algorithm is able to produce accurate results comparable to those obtained with SVM employing only the pairwise models learned in the construction of the hierarchy.

The data sets used in the experiments are described in Table 2. There are data sets with a greater number of examples than features, and vice versa. The first group is formed by data sets obtained from the UCI repository [2]. We selected those data sets that fulfill the following rules: continuous or ordinal attribute values, no more than 40 attributes, no more than 10,000 examples, while excluding data sets with missing values and with less than four classes. The second group is composed of the data sets used in [30]. These comprise seven data sets whose goal is to classify cancer patients from gene expressions captured by microarrays.

No results of $k$-means are shown as in this experiment we try to learn to distinguish all the classes (multi-class task) without grouping any of them.

Following [15], a two-step statistical test procedure was carried out. The first step consists of a Friedman test of the null hypothesis that all approaches perform equally. In the case of this hypothesis being rejected, a Nemenyi test is then conducted to compare the methods in a pairwise way. Both tests are based on the average of the ranks. The 0/1 loss comparison includes 5 algorithms over 18 data sets; so the critical difference (CD) at a significance level of $10 \%$ in the Nemenyi test is 1.2960. In the $P D$ comparison, only four algorithms were tested over the same data sets; in this case, the CD at the same significance level is 0.9859 .

Table 3 shows the $0 / 1$ loss results obtained in the experiments. As can be seen, SVM is the best method and DB2 is the worst, whereas PSMT produces error rates comparable to those obtained by SVM. DSVM and SMT provide similar results. To

Table 2

Description of the data sets used in the experiments. These are divided in two parts. The first is a collection of non-linearly separable classification tasks taken from the UCI repository [2]. The second part consists of cancer data sets that were previously used in [30].

\begin{tabular}{lcrr}
\hline Data set & \# Classes & \# Examples & \# Features \\
\hline Zoo & 7 & 101 & 16 \\
Glass & 6 & 214 & 9 \\
Ecoli & 8 & 336 & 7 \\
Dermatology & 6 & 366 & 33 \\
Vehicle & 4 & 846 & 18 \\
Vowel & 11 & 990 & 11 \\
Led & 10 & 1000 & 7 \\
Yeast & 10 & 1484 & 8 \\
Car & 4 & 1728 & 6 \\
Image & 7 & 2310 & 19 \\
Landsat & 6 & 6435 & 36 \\
Brain & 5 & 42 & 5597 \\
Lymphoma & 3 & 62 & 4026 \\
Srbct & 4 & 63 & 2308 \\
Stanford & 14 & 261 & 7452 \\
9 Tumors & 9 & 60 & 7131 \\
11 Tumors & 11 & 174 & 12,533 \\
14 Tumors & 14 & 190 & 16,063 \\
\hline
\end{tabular}


Table 3

Error rate of all methods. The number in parenthesis after the error rate is the rank of the method on the corresponding data set. The average rank is the average of the ranks across all data sets.

\begin{tabular}{|c|c|c|c|c|c|c|c|c|c|c|}
\hline \multirow[t]{2}{*}{ Data set } & \multicolumn{2}{|l|}{ SVM } & \multicolumn{2}{|c|}{ DSVM } & \multicolumn{2}{|l|}{ DB2 } & \multicolumn{2}{|l|}{ SMT } & \multicolumn{2}{|c|}{ PSMT } \\
\hline & $0 / 1$ & Rank & $0 / 1$ & Rank & $0 / 1$ & Rank & $0 / 1$ & Rank & $0 / 1$ & Rank \\
\hline Zoo & 5.0 & $(3.5)$ & 4.5 & $(2.0)$ & 5.5 & $(5.0)$ & 5.0 & $(3.5)$ & 4.0 & $(1.0)$ \\
\hline Glass & 33.2 & $(1.0)$ & 35.8 & $(3.0)$ & 36.2 & $(4.0)$ & 37.4 & $(5.0)$ & 33.9 & $(2.0)$ \\
\hline Ecoli & 11.8 & $(1.0)$ & 14.0 & $(4.0)$ & 14.3 & $(5.0)$ & 13.7 & $(3.0)$ & 11.9 & $(2.0)$ \\
\hline Dermatology & 3.5 & $(2.5)$ & 4.4 & $(5.0)$ & 3.4 & $(1.0)$ & 3.8 & $(4.0)$ & 3.5 & $(2.5)$ \\
\hline Vehicle & 20.3 & $(2.0)$ & 20.6 & (3.5) & 20.7 & $(5.0)$ & 20.6 & $(3.5)$ & 20.2 & $(1.0)$ \\
\hline Vowel & 19.2 & $(1.0)$ & 26.8 & $(3.0)$ & 28.3 & $(4.0)$ & 29.7 & $(5.0)$ & 21.8 & $(2.0)$ \\
\hline Led & 27.7 & $(2.0)$ & 27.8 & $(3.0)$ & 29.1 & $(5.0)$ & 28.0 & $(4.0)$ & 27.4 & $(1.0)$ \\
\hline Yeast & 41.6 & $(2.0)$ & 42.8 & $(3.0)$ & 43.4 & $(4.0)$ & 43.4 & $(5.0)$ & 41.6 & $(1.0)$ \\
\hline Car & 14.3 & $(1.0)$ & 15.1 & $(2.0)$ & 16.4 & $(4.0)$ & 17.3 & $(5.0)$ & 15.3 & (3.0) \\
\hline Image & 4.1 & $(1.0)$ & 10.1 & $(4.0)$ & 11.4 & $(5.0)$ & 4.3 & $(3.0)$ & 4.2 & $(2.0)$ \\
\hline Landsat & 13.1 & $(1.0)$ & 15.0 & $(5.0)$ & 14.9 & $(4.0)$ & 13.2 & $(3.0)$ & 13.1 & $(2.0)$ \\
\hline Brain & 13.2 & $(1.5)$ & 14.6 & $(4.0)$ & 18.1 & $(5.0)$ & 13.3 & $(3.0)$ & 13.2 & (1.5) \\
\hline Lymphoma & 0.0 & (3.0) & 0.0 & $(3.0)$ & 0.0 & $(3.0)$ & 0.0 & $(3.0)$ & 0.0 & (3.0) \\
\hline Srbct & 1.6 & $(4.0)$ & 0.8 & (1.5) & 1.6 & $(4.0)$ & 0.8 & $(1.5)$ & 1.6 & (4.0) \\
\hline Stanford & 5.4 & $(2.0)$ & 6.1 & $(5.0)$ & 5.9 & $(3.5)$ & 5.9 & $(3.5)$ & 5.4 & $(1.0)$ \\
\hline 9 Tumors & 48.3 & $(4.0)$ & 45.8 & $(3.0)$ & 40.8 & $(1.0)$ & 44.2 & $(2.0)$ & 49.2 & $(5.0)$ \\
\hline 11 Tumors & 10.1 & $(3.0)$ & 9.8 & $(2.0)$ & 9.5 & $(1.0)$ & 10.1 & $(4.0)$ & 10.4 & $(5.0)$ \\
\hline 14 Tumors & 30.5 & $(4.0)$ & 31.6 & $(5.0)$ & 30.0 & $(3.0)$ & 28.7 & $(1.0)$ & 29.5 & $(2.0)$ \\
\hline Avg. rank & & $(2.2)$ & & $(3.4)$ & & (3.7) & & $(3.4)$ & & $(2.3)$ \\
\hline
\end{tabular}

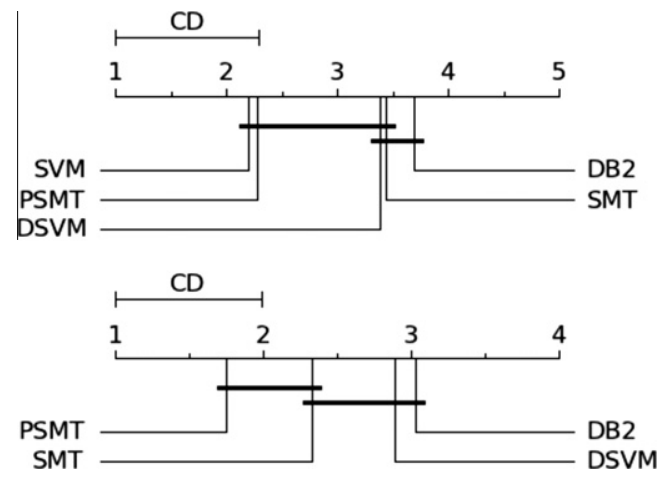

Fig. 4. Comparison of all algorithms vs one another using the Nemenyi test. Groups of classifiers that are not significantly different (at $p=0.1$ ) are connected. Above, comparison of error rates and below, comparison of prediction distances.

analyze the results in more depth, the Friedman test suggests that there are significant differences between the methods, while the Nemenyi test indicates that SVM is significantly better than the other algorithms, except for PSMT (see Fig. 4, top). No significant differences can be observed, however, between DB2, DVSM and SMT.

As mentioned previously, the quality of the hierarchies can be measured by the PD. We can see from Table 4 that PSMT achieves the best results, followed by SMT. DB2 and DSVM perform worse, so the distance in the hierarchy between predictions and the true class is bigger. In this case, the Nemenyi test shows us that PSMT is significantly better than DSVM and DB2 (see Fig. 4, bottom).

\subsection{Population genetics applications}

The aim of this section is to show that our approach (PSMT) is able to build meaningful hierarchical clusters of classes which are better than those obtained by other approaches and that it can be very useful to solve population genetics applications. We devote one section to each of the three applications addressed in this paper. We shall describe the main results and draw some useful conclusions for these applications.

In this experiment, we used all the clustering algorithms presented previously except SMT, because, as was shown in the previous section, PSMT obtains the same hierarchical clusterings, but better classifiers. The goal is to compare very different approaches: a clustering method without any structure (k-means), a top-down binary tree (DB2), a centroids-based hierarchical clustering (DSVM) and a pairwise-based hierarchical clustering (PSMT).

As we wish to explain the results obtained by the hierarchical classifiers learned by PSMT using concrete dendrograms of classes, first of all the clustering of classes is obtained for all the methods considered using the complete data set of exam- 
Table 4

Prediction Distance of all methods. The number in parenthesis is the rank of the method on the corresponding data set. The average rank is the average of the ranks across all data sets.

\begin{tabular}{|c|c|c|c|c|c|c|c|c|}
\hline \multirow[t]{2}{*}{ Data set } & \multicolumn{2}{|l|}{ DSVM } & \multicolumn{2}{|l|}{ DB2 } & \multicolumn{2}{|l|}{ SMT } & \multicolumn{2}{|l|}{ PSMT } \\
\hline & $P D$ & Rank & $P D$ & Rank & $P D$ & Rank & $P D$ & Rank \\
\hline Zoo & 0.275 & $(3.0)$ & 0.284 & $(4.0)$ & 0.179 & $(2.0)$ & 0.134 & $(1.0)$ \\
\hline Glass & 1.129 & $(2.0)$ & 1.141 & $(3.0)$ & 1.150 & $(4.0)$ & 0.986 & $(1.0)$ \\
\hline Ecoli & 0.387 & $(3.0)$ & 0.425 & $(4.0)$ & 0.378 & $(2.0)$ & 0.292 & $(1.0)$ \\
\hline Dermatology & 0.104 & $(4.0)$ & 0.076 & $(1.0)$ & 0.093 & $(3.0)$ & 0.079 & $(2.0)$ \\
\hline Vehicle & 0.482 & $(4.0)$ & 0.479 & $(2.0)$ & 0.480 & $(3.0)$ & 0.462 & $(1.0)$ \\
\hline Vowel & 1.135 & $(2.0)$ & 1.371 & $(4.0)$ & 1.345 & $(3.0)$ & 0.825 & $(1.0)$ \\
\hline Led & 1.195 & $(3.0)$ & 1.358 & $(4.0)$ & 1.186 & $(1.0)$ & 1.189 & $(2.0)$ \\
\hline Yeast & 1.434 & $(3.0)$ & 1.510 & $(4.0)$ & 1.367 & $(2.0)$ & 1.286 & $(1.0)$ \\
\hline Car & 0.475 & $(4.0)$ & 0.412 & $(2.0)$ & 0.416 & $(3.0)$ & 0.346 & $(1.0)$ \\
\hline Image & 0.381 & $(3.0)$ & 0.522 & $(4.0)$ & 0.113 & $(2.0)$ & 0.106 & $(1.0)$ \\
\hline Landsat & 0.583 & $(3.0)$ & 0.646 & $(4.0)$ & 0.405 & $(2.0)$ & 0.405 & $(1.0)$ \\
\hline Brain & 0.561 & $(3.0)$ & 0.808 & $(4.0)$ & 0.512 & $(2.0)$ & 0.482 & $(1.0)$ \\
\hline Lymphoma & 0.000 & $(2.5)$ & 0.000 & $(2.5)$ & 0.000 & $(2.5)$ & 0.000 & $(2.5)$ \\
\hline Srbct & 0.017 & $(1.0)$ & 0.047 & $(3.0)$ & 0.025 & $(2.0)$ & 0.048 & $(4.0)$ \\
\hline Stanford & 0.270 & $(1.0)$ & 0.343 & $(4.0)$ & 0.316 & $(3.0)$ & 0.285 & $(2.0)$ \\
\hline 9 Tumors & 2.308 & $(2.5)$ & 2.242 & $(1.0)$ & 2.308 & $(2.5)$ & 2.542 & $(4.0)$ \\
\hline 11 Tumors & 0.549 & $(4.0)$ & 0.485 & $(1.0)$ & 0.521 & $(2.0)$ & 0.545 & $(3.0)$ \\
\hline 14 Tumors & 1.903 & $(4.0)$ & 1.874 & $(3.0)$ & 1.529 & $(1.0)$ & 1.611 & $(2.0)$ \\
\hline Avg. rank & & $(2.9)$ & & $(3.0)$ & & $(2.3)$ & & $(1.8)$ \\
\hline
\end{tabular}

ples. We then estimate the different classification errors (0/1 loss and $P D$, except for k-means, as it is not applicable) by means of a fivefold cross-validation repeated twice. If we had included the construction of the clustering in the cross-validation process, the clusterings could be different in each execution. Therefore, the classification errors thus obtained would not correspond to a particular clustering. Although this could be seen as an optimistic estimation, other experiments carried out showed that both methods produced almost identical results. In any case, all the algorithms considered use the same procedure.

\subsubsection{Iberian roe deer (C. capreolus)}

Royo et al., see [29], studied 109 Iberian roe deer individuals corresponding to 9 Spanish populations. Individuals were sampled at locations that were expected to have acted as refugia for the species during the decrease in population size in the 20th century. Samples were analyzed by means of 10 microsatellites. Using these microsatellites, correspondence analysis and molecular coancestry information revealed high molecular differentiation among Northwestern $(\{1,5,7,9\})$ and Central-Southern $(\{3,4,8\})$ Spanish roe deer populations, with two Pyrenean populations $(\{2,6\})$ being characterized as non-local, reintroduced populations.

Analyzing the hierarchical clustering obtained by PSMT (Fig. 2) it seems clear that there are two main groups corresponding to the two main regions described in [29]. The left cluster $\{1,5,7,9,2,6\}$ corresponds to Northwestern populations (with a further subdivision including the Pyrenean populations: 2 and 6), while the right cluster $\{3,4,8\}$ is formed by CentralSouthern populations. In this case, the clustering obtained is almost geographical, but well explained according to the between-populations genetic identities found in the original genetic study cited previously. In fact, the classifier for these two groups has a low $0 / 1$ loss, considering that the original task is quite difficult.

Fig. 3 shows the performance of the different algorithms when the number of clusters increases. It can be seen that with four clusters, PSMT obtains acceptable results: less than $11 \%$ in $0 / 1$ loss and less than 0.3 in PD. The clusters are the following: (i) $\{1,5,7,9\}$ includes Northwestern roe deer, (ii) $\{2,6\}$ animals from the Pyrenees area, (iii) $\{3,4\}$ Southern populations, and (iv) $\{8\}$ animals from the Iberian branch.

\subsubsection{Eurasian otter (L. lutra) in Britain}

In [13], the authors assessed the effects of gene flow and population fragmentation on the genetic structure in British populations of the Eurasian otter. The analysis was based on genotypes consisting of 12 microsatellites from 618 individuals belonging to 17 different populations. The main goal of the paper was to compare the genetic makeup of the continuous otter population in Scotland with respect those in Wales and South West England. Otters in southern Britain contained significantly lower levels of microsatellite polymorphism than otters in Scotland, and the population in the western peninsula of SW England was genetically distinct. Interestingly, the Scottish otter population showed a significant genetic structure (Northern Isles, North Scotland and South Scotland), which was more likely due to restricted contemporary gene flow than to historical fluctuations in the size of subpopulations. 

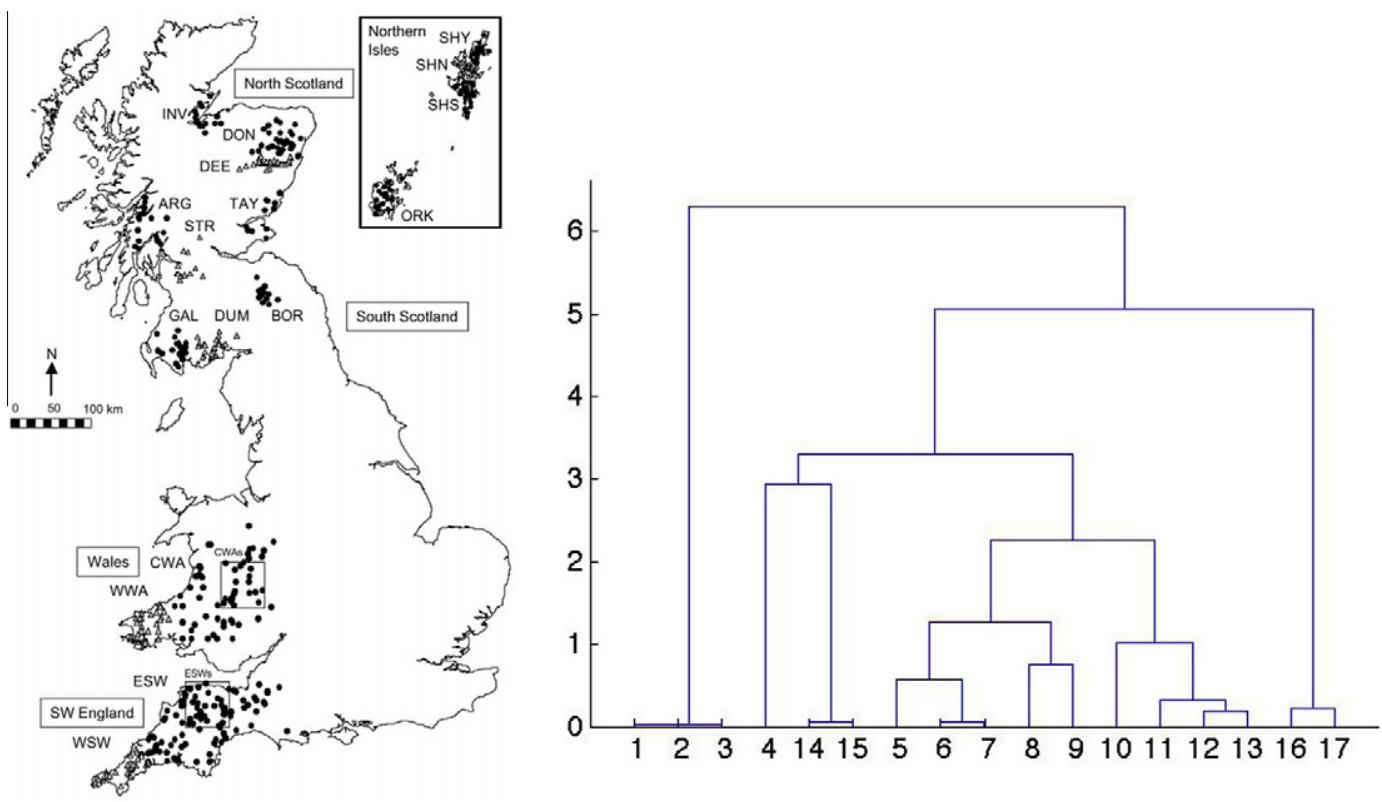

Fig. 5. Geographical distribution of populations in the Otters data set. Northern Isles: (1) Shetland North (SHN), (2) Shetland South (SHS), (3) Shetland Yell (SHY), (4) Orkney (ORK); North Scotland: (5) Inverness (INV), (6) R. Don (DON), (7) R. Dee (DEE), (8) Tayside (TAY), (9) Argyll (ARG), (10) Strathclyde (STR); South Scotland: (11) Borders (BOR), (12) Dumfries (DUM), (13) Galloway (GAL); Wales: (14) Central (CWA), (15) West (WWA); and SW England: (16) East (ESW), (17) West (WSW). The map was taken from [13].

Fig. 5 shows the hierarchical clustering obtained by PSMT and the geographical layout of the different populations. It can be seen that the algorithm obtains a geographically coherent hierarchy, creating groups with otters from the Northern Isles, North Scotland, South Scotland, Wales and SW England. At first sight, it may seem strange that ORK, a Northern Isles population, appears close to Welsh populations, but this is not so strange given that [13] showed that there are no significant differences in levels of microsatellite polymorphism between these populations. Although the connection between this pair of breeds is supported by the bibliography, we can appreciate that this connection is produced at a high distance. A similar situation occurs with STR, a North Scotland population, which is placed close to South Scotland populations; once again, significant differences were not found between these populations in [13]. However, in this case our method assesses a lower distance to the node separating the STR and the South Scotland otter populations. The lack of differences between STR and the other members of its cluster may be explained by a continuous between-populations gene flow in the area of the source of the rivers flowing to these areas. Once more, our method automatically obtains a hierarchical clustering that is supported by previous studies in the field of population genetics.

An analysis of how the algorithms perform when the number of clusters is increased can be seen in Fig. 6 . With 5 clusters, we obtain $1 \%$ in $0 / 1$ loss and 0.03 in $P D$. The clusters are the following: (i) $\{1,2,3\}$ Shetland populations; (ii) \{4\} Orkney otters; (iii) $\{14,15\}$ Wales populations; (iv) $\{5,6,7,8,9,10,11,12,13\}$ Scotland populations; and (v) $\{16,17\}$ SW England otters. If we wish to descend one step in the hierarchy, we will have to split the fourth cluster in two: $\{5,6,7,8,9\}$ North Scotland populations, and $\{10,11,12,13\}$ South Scotland population plus STR. With these six clusters, the $0 / 1$ loss increases to $2.5 \%$ and the $P D$ to 0.07 .

\subsubsection{African cattle structure}

Hanotte et al., see [20], genotyped 15 microsatellite loci on 50 local African cattle breeds fully representative of presentday African cattle stock (mainly populations with a different admixture of B. taurus and B. indicus, known as 'sanga'), and on 5 European B. taurus and 3 Asian B. indicus cattle breeds, used as outgroups, in order to ascertain the genetic signatures of their origins, spread and differentiation. The hypotheses tested were: (a) an expansion of the 'original' West African cattle southwards (which occurred during the pastoralist migrations of the Middle Ages that eventually invaded present-day South Africa); (b) an intense B. indicus introgression into the 'original' African taurine cattle from the Horn and the East Coast of Africa to Sub-Saharan West Africa and to South Africa via Tanzania and Malawi; and (c) a recent genetic influence of European $B$. taurus cattle.

The hierarchy obtained by the PSMT algorithm is presented in Fig. 7. To analyze the hierarchy, we break up the hierarchical clustering to obtain five groups of populations. From left to right, the first group is composed of $\{56,57,58\}$ Asian breeds, all of which are $B$. indicus. The second group contains 27 breeds: 17 B. indicus and 10 B. taurus. This coincides with the findings by [20], as these $10 \mathrm{~B}$. taurus breeds have the highest estimated proportion of Asian zebu admixture (see Sup- 

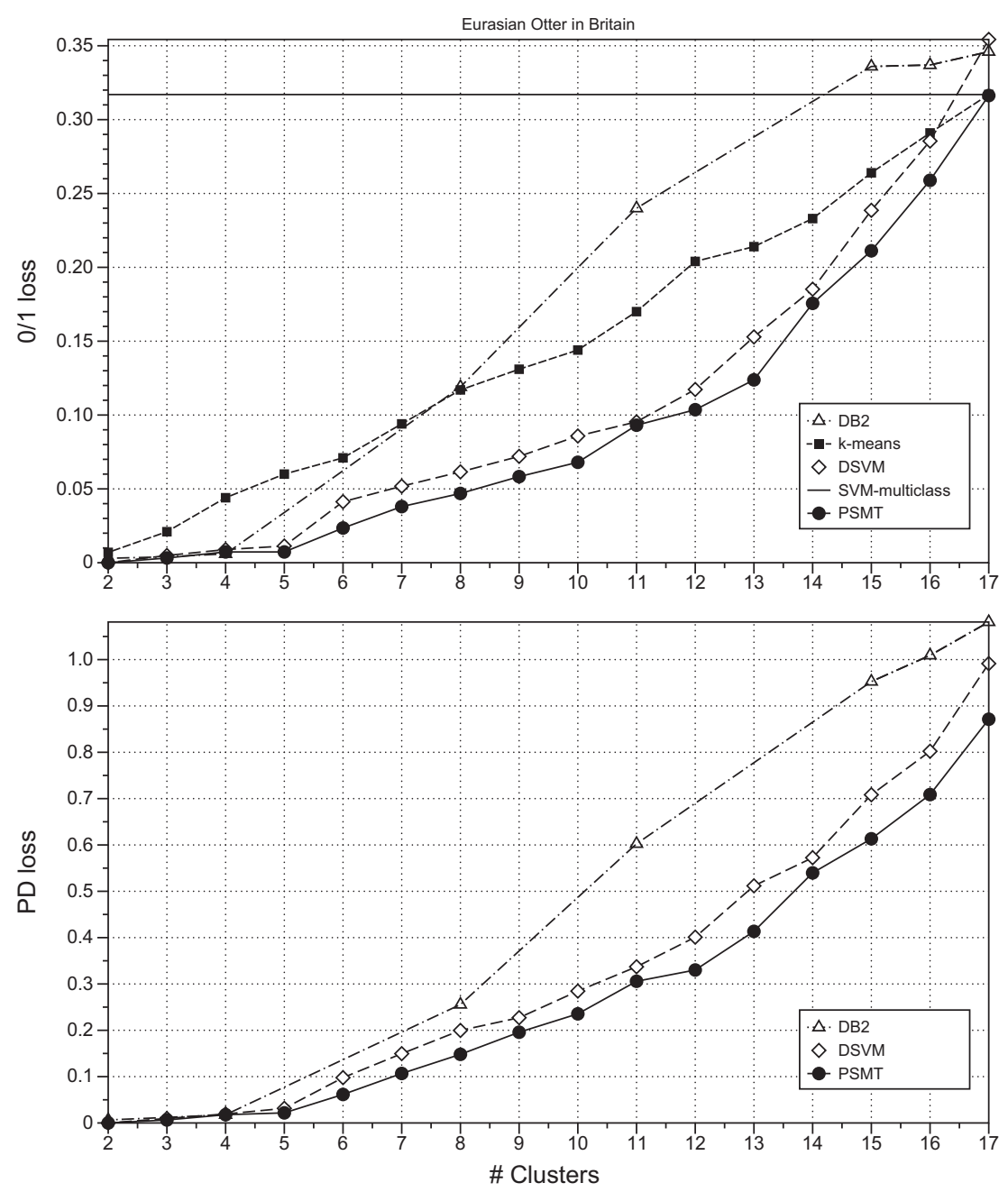

Fig. 6. Results in the Otters data set. At the top, the graph of the $0 / 1$ loss for each number of clusters. At the bottom, the Prediction Distance loss only for the DSVM, PSMT and DB2 methods.

plementary Data in [20]). The third group has 2 B. indicus and 16 B. taurus breeds. According to the Supplementary Data in [20], the two B. indicus breeds have the lowest estimated proportion of Asian zebu admixture. The fourth group is composed of $\{11,46,36,22,35\}$ breeds, all of which are $B$. taurus. Finally, the last group contains $\{13,53,52,54,55\}$ breeds, namely four European breeds and the Moroccan Blonde breed (highly influenced by European cattle), all of which are B. taurus.

Fig. 8 shows the performance of the algorithms when the number of clusters increases. It can be seen that, for five clusters, the PSMT obtains $12.6 \%$ of $0 / 1$ loss and 0.53 of $P D$. The higher the number of clusters assumed, the higher the betweenalgorithms differences in performance.

\section{Conclusions}

We have developed a new methodology to deal with data in the field of population genetics. Starting from the genetic description of a set of individuals belonging to a limited number of populations (e.g. different species or breeds), our system, PSMT, is able to produce: (1) a hierarchical clustering of populations and (2) a model for a hierarchical classification of individuals. Both the hierarchical clustering and the classifier can be broken up at different levels to yield different clusterings of the set of classes and different classifiers. We have shown the usefulness of PSMT, presenting the experimental results achieved with three interesting applications in the field of population genetics.

The paper also presents a new method for the classification stage of the hierarchical model in a multi-class learning task. This method is faster than the previous one and obtains better results in the data sets used in the experiments: 11 UCI data sets and 7 data sets whose goal is to classify cancer patients from gene expressions captured by microarrays. We compare 


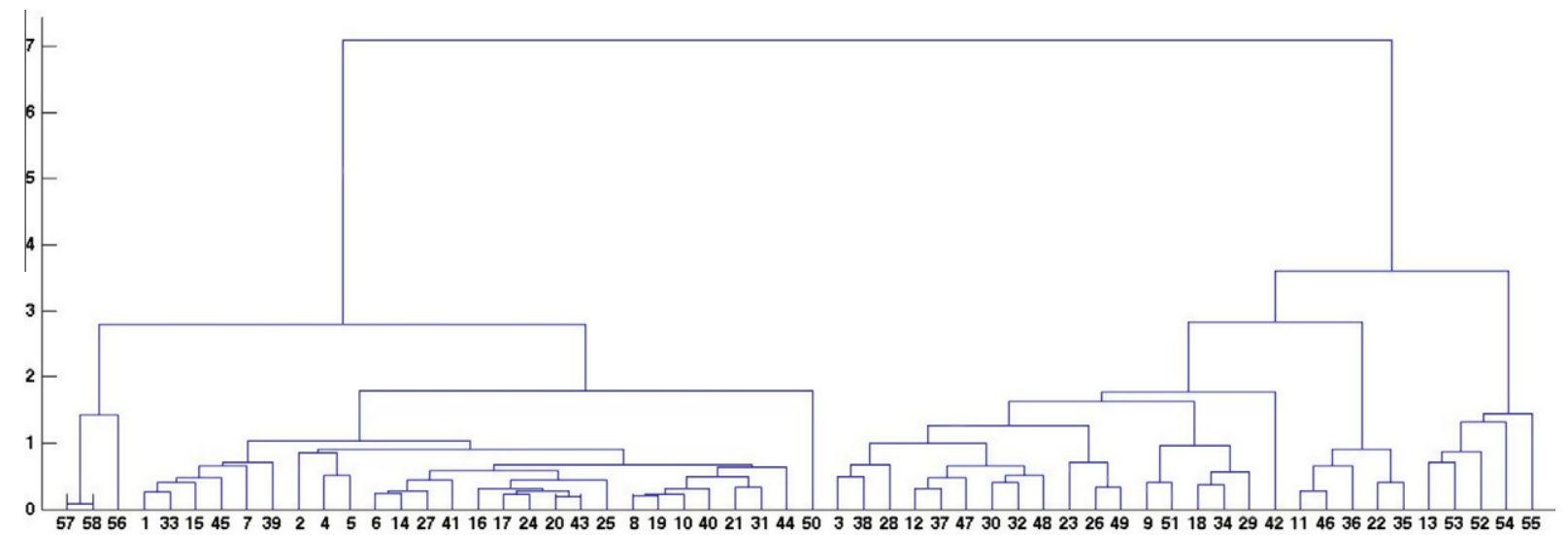

Fig. 7. Name of the breeds in the African cattle data set. In parenthesis, the number of the breed and a letter indicating whether it is $B$. taurus (T) or B. indicus (I). (1T) abigar, (2T) afar, (3T) africaner, (4I) angoni, (5T) ankole, (6T) arado, (7I) arashi, (8I) arsi, (9T) baladi, (10I) bale, (11T) baoule, (12T) barotse, (13T) blonde, (14I) boran eth, (15I) butana foya, (16T) danakil, (17T) fogera, (18I) gobra, (19I) highland zebu, (20T) horro, (21I) iringa red, (22T) kapsiki, (23T) kavango, (24I) kavirondo, (25I) kenyan boran, (26T) kigezi, (27I) kilimanjaro, (28T) koakoland, (29T) kuri, (30T) landim, (31I) malawi zebu, (32T) mashona, (33I) maure, (34I) mbororo, (35T) namchi, (36T) ndama, (37T) nguni, (38T) nkone, (39T) nuba, (40I) ogaden, (41I) orma boran, (42T) pedi, (43T) raya azebu, (44T) sheko, (45I) sokoto gudali, (46T) somba, (47T) tonga, (48T) tuli, (49T) watusi, (50T) zebu malagasy, (51T) anatblack, (52T) charolais, (53T) friesian, (54T) jersey, (55T) retinta, (56I) shhiwal, (57I) ongolenew, (58I) nelore ongole. These populations are from: Africa (1-50), Europe (51-55), and Asia (56-58).
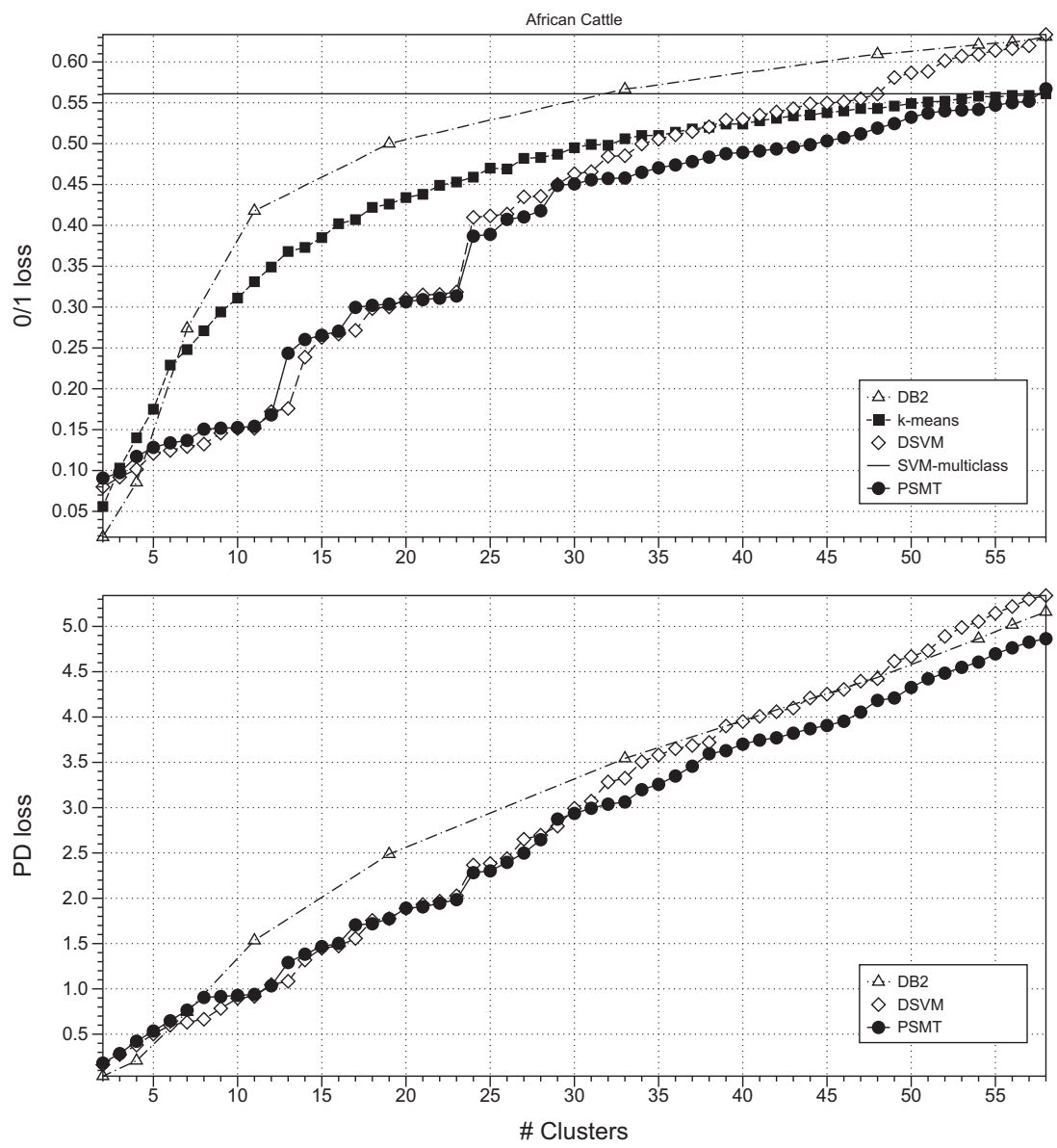

Fig. 8. Results in the African cattle data set. At the top, the graph of the 0/1 loss for each number of clusters. At the bottom, the Prediction Distance loss only for the DSVM, PSMT and DB2 methods. 
our algorithm with five other algorithms, concluding that PSMT performs well both as a multi-class classifier and as a clustering algorithm. Moreover, the proposed method is computationally as efficient as the well-known one-vs-one multi-class SVM.

\section{Acknowledgements}

The authors are indebted to Dr. Olivier Hanotte for kindly providing the African cattle genetic dataset used in this work. This research has been partially supported by Spanish Ministerio de Ciencia e Innovación (MICINN) Grants TIN2008-06247 and TIN2011-23558.

\section{References}

[1] A. Ahmadi, S. Omatu, T. Fujinaka, T. Kosaka, Improvement of reliability in banknote classification using reject option and local PCA, Information Sciences 168 (2004) 277-293.

[2] A. Asuncion, D. Newman, UCI Machine Learning Repository, School of Information and Computer Sciences, University of California, Irvine, California, USA, 2007.

[3] R.C. Barros, D.D. Ruiz, M.P. Basgalupp, Evolutionary model trees for handling continuous classes in machine learning, Information Sciences 181 (2011) 954-971.

[4] P. Bartlett, M. Wegkamp, Classification with a reject option using a hinge loss, Journal of Machine Learning Research 9 (2008) $1823-1840$.

[5] K. Benabdeslem, Y. Bennani, Dendogram based SVM for multi-class classification, Journal of Computing and Information Technology 14 (2006) $283-289$.

[6] B.E. Boser, I.M. Guyon, V.N. Vapnik, A training algorithm for optimal margin classifiers, in: Proceedings of the Fifth Annual Workshop on Computational Learning Theory, COLT '92, ACM, New York, NY, USA, 1992, pp. 144-152.

[7] N. Cesa-Bianchi, C. Gentile, L. Zaniboni, Incremental algorithms for hierarchical classification, Journal of Machine Learning Research 7 (2006) $31-54$.

[8] C. Chow, On optimum recognition error and reject tradeoff, IEEE Transactions on Information Theory 16 (1970) 41-46.

[9] G. Corani, M. Zaffalon, Learning reliable classifiers from small or incomplete data sets: the Naïve credal classifier 2, Journal of Machine Learning Research 9 (2008) 581-621.

[10] J.J. del Coz, J. Díez, A. Bahamonde, Learning nondeterministic classifiers, Journal of Machine Learning Research 10 (2009) $2273-2293$.

[11] K. Crammer, Y. Singer, On the algorithmic implementation of multiclass kernel-based vector machines, Journal of Machine Learning Research 2 (2001) 265-292.

[12] N. Cristianini, J. Shawe-Taylor, An Introduction to Support Vector Machines and Other Kernel-based Learning Methods, Cambridge University Press, 2000.

[13] J. Dallas, F. Marshall, S. Piertney, P. Bacon, P. Racey, Spatially restricted gene flow and reduced microsatellite polymorphism in the Eurasian otter Lutra lutra in Britain, Conservation Genetics 3 (2002) 15-29.

[14] O. Dekel, J. Keshet, Y. Singer, Large margin hierarchical classification, in: Proceedings of the Twenty-First International Conference on Machine learning, ICML '04, ACM, New York, NY, USA, 2004, pp. 209-216.

[15] J. Demšar, Statistical comparisons of classifiers over multiple data sets, Journal of Machine Learning Research 7 (2006) 1-30.

[16] J. Díez, J. Coz, A. Bahamonde, O. Luaces, Soft margin trees, in: European Conference on Machine Learning and Principles and Practice of Knowledge Discovery in Databases (ECML PKDD ’09), Springer-Verlag, 2009, pp. 302-314.

[17] J. Díez, J.J. del Coz, A. Bahamonde, A semi-dependent decomposition approach to learn hierarchical classifiers, Pattern Recognition 43 (2010) 3795-3804.

[18] A.A. Freitas, D.C. Wieser, R. Apweiler, On the importance of comprehensible classification models for protein function prediction, IEEE/ACM Transactions on Computational Biology and Bioinformatics 7 (2010) 172-182.

[19] J. Friedman, Another Approach to Polychotomous Classification, Technical Report, Department of Statistics, Stanford University, 1996.

[20] O. Hanotte, D.G. Bradley, J.W. Ochieng, Y. Verjee, E.W. Hill, J.E.O. Rege, African pastoralism: genetic imprints of origins and migrations, Science 296 (2002) 336-339.

[21] J.A. Hartigan, Clustering Algorithms, John Wiley \& Sons, Inc., New York, NY, USA, 1975.

[22] C.C. Hsu, C.L. Chen, Y.W. Su, Hierarchical clustering of mixed data based on distance hierarchy, Information Sciences 177 (2007) $4474-4492$.

[23] J. Huysmans, K. Dejaeger, C. Mues, J. Vanthienen, B. Baesens, An empirical evaluation of the comprehensibility of decision table, tree and rule based predictive models, Decision Support Systems 51 (2011) 141-154.

[24] U. Kreßel, Pairwise classification and support vector machines, in: B. Schölkopf, C.J.C. Burges, A.J. Smola (Eds.), Advances in Kernel Methods - Support Vector Learning, MIT Press, Cambridge, MA, 1999, pp. 255-268.

[25] J.Z. Lai, T.J. Huang, An agglomerative clustering algorithm using a dynamic k-nearest-neighbor list, Information Sciences 181 (2011) $1722-1734$.

[26] H. Lei, V. Govindaraju, Half-against-half multi-class support vector machines, in: 6th International Workshop on Multiple Classifier Systems, Springer, 2005, pp. 156-164.

[27] J.K. Pritchard, M. Stephens, P. Donnelly, Inference of population structure using multilocus genotype data, Genetics 155 (2000) $945-959$.

[28] J. Rousu, C. Saunders, S. Szedmak, J. Shawe-Taylor, Kernel-based learning of hierarchical multilabel classification models, Journal of Machine Learning Research 7 (2006) 1601-1626.

[29] L.J. Royo, G. Pajares, I. Álvarez, I. Fernández, F. Goyache, Genetic variability and differentiation in spanish roe deer (Capreolus capreolus): A phylogeographic reassessment within the european framenwork, Molecular Phylogenetics and Evolution 42 (2007) 47-61.

[30] R. Tibshirani, T. Hastie, Margin trees for high-dimensional classification, Journal of Machine Learning Research 8 (2007) 637-652.

[31] V. Vapnik, Statistical Learning Theory, John Wiley \& Sons, Inc., New York, NY, USA, 1998.

[32] V. Vural, J.G. Dy, A hierarchical method for multi-class support vector machines, in: ICML '04: Proceedings of the Twenty-First International Conference on Machine Learning, ACM, New York, NY, USA, 2004, pp. 105-112.

[33] T. Wu, C. Lin, R. Weng, Probability estimates for multi-class classification by pairwise coupling, Journal of Machine Learning Research 5 (2004) 975-1005.

[34] C. Zhong, D. Miao, P. Fränti, Minimum spanning tree based split-and-merge: a hierarchical clustering method, Information Sciences 181 (2011) 3397-3410. 